\title{
Baryonic pollution in gamma-ray bursts: The case of a magnetically driven wind emitted from a disk orbiting a stellar mass black hole
}

\author{
F. Daigne ${ }^{1, \star}$ and R. Mochkovitch ${ }^{2}$ \\ 1 Max-Planck-Institut für Astrophysik, Karl-Schwarzschild-Str 1, Postfach 1317, 85741 Garching bei München, \\ Germany \\ 2 Institut d'Astrophysique de Paris, 98bis Bld. Arago, 75014 Paris, France
}

Received 15 October 2001 / Accepted 25 February 2002

\begin{abstract}
Most models for the central engine of gamma-ray bursts involve a stellar mass black hole surrounded by a thick disk formed after the merging of a system of compact objects or the collapse of a massive star. Energy released from the accretion of disk material by the black hole or from the rotation of the hole itself extracted by the Blandford-Znajek mechanism powers a relativistic wind along the system axis. Lorentz factors of several hundreds are needed to solve the compactness problem in the wind which implies the injection of a tremendous power into a very small amount of matter. The Blandford-Znajek mechanism, where the outflow follows magnetic field lines anchored to the black hole is probably the best way to prevent baryonic pollution and can even initially produce a purely leptonic wind. In this paper we rather study the wind emitted from the inner part of the disk where the risk of baryonic pollution is much larger since the outflow originates from high density regions. We show that the baryonic load of this wind sensitively depends on the disk temperature and magnetic field geometry and that the outflow can become ultra-relativistic (Lorentz factor $\Gamma>100$ ) under quite restrictive conditions only. Conversely, if $\Gamma$ remains of the order of unity the dense wind emitted from the inner disk could help to confine the central jet but may also represent a source of baryon contamination for the Blandford-Znajek mechanism.
\end{abstract}

Key words. gamma rays: bursts - accretion: accretion disks - magnetohydrodynamics (MHD) - neutrinos relativity

\section{Introduction}

The discovery of the first optical counterparts to gammaray bursts (hereafter GRBs) in 1997 (van Paradijs et al. 1997) has shown that most (if not all) GRBs are located at cosmological distances. About 20 redshifts have now been measured from $z=0.43$ for GRB 990712 to $z=4.5$ for GRB 000131 (with however the peculiar case of GRB 980425 which appears to be associated to a nearby type Ic supernova at $z=0.01$; Galama et al. 1998). The energy radiated by these cosmological GRBs in the BATSE range $(20-1000 \mathrm{keV})$ goes from $5 \times 10^{51}$ ergs for GRB 970228 and GRB 980613 (at $z=0.695$ and 1.096) to $2 \times 10^{54}$ ergs for GRB 990123 (at $z=1.6$ ) assuming isotropic emission. After correction for beaming, the true energy output appears to be less scattered, clustered around $E_{\gamma} \sim 5 \times 10^{50}$ ergs (Djorgovski et al. 2001). Among

Send offprint requests to: F. Daigne,

e-mail: daigne@discovery.saclay.cea.fr

* Present address: Service d'Astrophysique, CEA/Saclay, 91191 Gif-sur-Yvette Cedex, France the sources which have been proposed to explain such a huge release of energy in a time of seconds, the most popular are mergers of compact objects (neutron star binaries or neutron star - black hole systems) (Narayan et al. 1992; Meszaros \& Rees 1992b; Mochkovitch et al. 1993) or massive stars which collapse to a black hole (collapsars) (Woosley 1993; Paczynski 1998). In all these cases, the resulting configuration is a stellar mass black hole surrounded by a thick torus made of stellar debris or of infalling stellar material partially supported by centrifugal forces. The location of the detected optical counterparts well inside their host galaxies and often associated with regions of star formation appears to favor the collapsar scenario (Paczynski 1998; Owens et al. 1998; Klose et al. 2000). Double neutron star or neutron star - black hole mergers should generally be observed at the periphery of the host galaxy due to the long delay before coalescence and the large velocity imparted to these systems by two successive supernova explosions. They can however still be invoked in the case of shorts bursts, for which no optical counterpart has been detected. 
If black hole + thick disk configurations are indeed at the origin of GRBs, the released energy will ultimately come from the accretion of disk material by the black hole or from the rotational energy of the hole itself extracted by the Blandford-Znajek mechanism (Blandford \& Znajek 1977). In a first step, the energy must be injected into a relativistic wind whose existence has been directly inferred from the observations of radio scintillation in GRB 970508 (Waxman et al. 1998) and which is also needed to avoid photon-photon annihilation and the resulting compactness problem (Baring \& Harding 1997). The second step consists of the conversion of a fraction of the wind kinetic energy into gamma-rays via the formation of shocks, probably inside the wind itself (Rees \& Meszaros 1994). These internal shocks can be expected if the source generate a highly non uniform distribution of Lorentz factor so that rapid layers of the wind will catch up with slower ones at large relative velocities. In the last step, the wind is decelerated when it interacts with the interstellar or circumstellar medium and the resulting (external) shock is responsible for the afterglow observed in the X-ray, optical and radio bands (Meszaros \& Rees 1997).

The physics of the afterglow is probably the best understood since most afterglow properties can be interpreted in terms of solutions of the relativistic Sedov problem (Blandford \& McKee 1976) with synchrotron emission behind the shock (Sari et al. 1998). Models taking into account the beam geometry of the flow (Rhoads 1997) or different kinds of burst environments (constant density medium or stellar wind; Chevalier \& Li 2000) can be constructed to explain observed breaks in the lightcurves or the evolution of some radio afterglows.

More problems remain concerning the generation of gamma-rays in the relativistic wind. Instead of internal shocks, gamma-rays can also be emitted during the early evolution of the external shock with however the difficulty to explain in this case the highly variable temporal profiles of observed bursts (Sari et al. 1998 see however Dermer \& Mitman 1999). Models of bursts produced by internal shocks are found to be in reasonable agreement with the observations (Kobayashi et al. 1997; Daigne \& Mochkovitch 1998, 2000) even if the process which produces the gamma-rays - synchrotron radiation or/and comptonization - remains uncertain due to problems encountered in fitting the low energy part of the spectra (Preece et al. 1998).

The origin of the relativistic wind is the more complex of the three steps. Several proposals have been made to explain the generation of this wind but few detailed calculations have been performed. If the burst energy comes from matter accretion by the black hole, the annihilation of neutrino-antineutrino pairs emitted by the hot disk could be a way to inject energy along the system axis, in a region which can be expected to be depleted in baryons due to the effect of centrifugal forces (Meszaros \& Rees 1992a; Mochkovitch et al. 1993). The low efficiency of this process requires high neutrino luminosities and therefore high accretion rates. In the merger case this can be achieved for short accretion timescales (Ruffert \& Janka 1999) and may explain short bursts. Conversely in the collapsar scenario, the larger mass reservoir allows the system to maintain high accretion rates for a longer time and could then also produce long bursts (MacFadyen \& Woosley 1999).

Another possibility is to suppose that disk energy is extracted by a magnetic field amplified by differential rotation up to very large values $\left(B \gtrsim 10^{15} \mathrm{G}\right)$. A magnetically driven wind could then be emitted from the disk with a fraction of the Poynting flux being eventually transferred to matter (Blandford \& Payne 1982). Such a mechanism operates in many classes of astrophysical objects (from T Tauri stars to AGN) but it is far from clear that it can work in the context of GRBs where final Lorentz factors of several hundreds are required. In a different version of the same idea, an early conversion of magnetic into thermal energy could occur through the reconnection of field lines above the plane of the disk in a region of rather low density (Narayan et al. 1992).

An alternative to accretion is to directly extract the rotational energy of the black hole via the Blandford-Znajek mechanism. The available power then depends on the rotation parameter $a$ of the hole and on the intensity of the magnetic field pervading the horizon. If $B \gtrsim 10^{15} \mathrm{G}$ and $a \sim 1$, the power available from the Blandford-Znajek mechanism can be larger than $10^{52} \mathrm{erg} \mathrm{s}^{-1}$ with a very limited contamination by baryons at the source since the field lines which guide the outflow are anchored to the black hole.

In this paper we rather concentrate on the wind which is emitted from the inner disk. We want to identify the key parameters which control its baryonic load and check whether it can reach large Lorentz factors or remains non relativistic. Our approach will be oversimplified in comparison to the complexity of the real problem so that our conclusions have to be considered as indicative only. In Sect. 2 we briefly discuss the structure of the disk + black hole configurations which are produced by NS $+\mathrm{NS}$ or $\mathrm{NS}+\mathrm{BH}$ mergers and in the collapsar scenario. We write in Sect. 3 the equations which govern the wind dynamics from the disk to the sonic point. They are solved in Sect. 4 to obtain the mass loss rate and the terminal Lorentz factor of the wind is estimated in Sect. 5. Our results are discussed in Sect. 6 which is also the conclusion.

\section{The structure of the disk}

Before writing the wind equations in the next section we first describe the black hole + disk configurations which are obtained in the case of the three most discussed GRB sources: NS + NS or BH + NS mergers and collapsars. We want to compare the black hole and disk masses, the hole rotation parameter $a$, estimate which fraction of the disk is optically thick to neutrinos and obtain the disk temperature. 

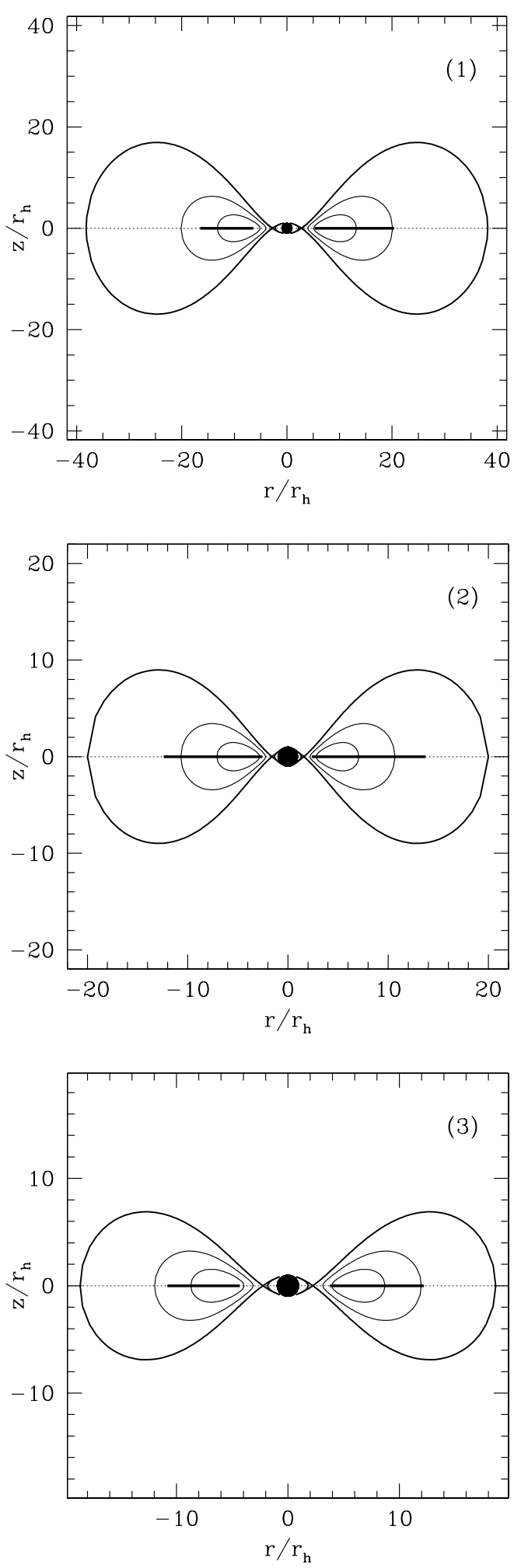

Fig. 1. Equilibrium models for thick accreting disks orbiting stellar mass black holes. The disks are supported by the pressure of relativistic degenerate electrons and the specific angular momentum is increasing outwards as $r^{0.2}$; case 1) Schwarzschild black hole of mass $M_{\mathrm{BH}}=2.5 M_{\odot}$ and disk mass $\left.M_{\mathrm{D}}=0.3 M_{\odot} ; 2\right)$ same $M_{\mathrm{BH}}$ and $M_{\mathrm{D}}$ and Kerr black hole with $a=0.8$; 3) $M_{\mathrm{BH}}=5 M_{\odot}, M_{\mathrm{D}}=0.5 M_{\odot}$ and $a=0.4$. In each model thick lines in the equatorial plane indicate the extension of the zone which is optically thick to neutrinos for $T_{\nu}=2$ (left) and 3 (right) $\mathrm{MeV}$.

\subsection{Mergers}

The coalescence of neutron stars has been studied by several groups mostly in the Newtonian and postNewtonian approximation (Davies et al. 1994; Ruffert et al. 1996; Calder et al. 1999; Rosswog et al. 2000; Faber et al. 2000) The resulting merged object obtained from two neutron stars of $1.4 M_{\odot}$ is made of a dense central core of $\sim 2.5 M_{\odot}$ in quasi-uniform rotation surrounded by a differentially rotating disk of $\sim 0.3 M_{\odot}$. In relativistic calculations, a black hole is directly formed during the merging event if the total mass of the system is 30 to $70 \%$ larger than the maximum rest mass of an isolated neutron star (Oohara \& Nakamura 1997). Due to the large angular momentum of the merger the rotation parameter $a=J c / G M_{\mathrm{BH}}^{2}$ of the newly formed black hole can easily exceed 0.5 .

In the case of $\mathrm{BH}+\mathrm{NS}$ mergers, the masses of the disk and black hole are in average larger than in NS + NS mergers. The hole rotation parameter depends on the fraction of neutron star material accreted by the black hole during the merging event. Janka et al. (1999) obtain disk masses between 0.3 and $0.7 M_{\odot}$ and rotation parameter between 0.1 and 0.5 for different assumptions regarding the relative masses and spins of the neutron star and the black hole.

To estimate the transparency of the disks to neutrinos we have computed the structure of the merged object with the self-consistent field method originally developed by Ostriker \& Mark (1968) using the approach of Hachisu (1986) to solve the Poisson equation. The equation of state in the disk corresponds to an ideal gas of ultra-relativistic electrons and the distribution of specific angular momentum is $j(r) \propto r^{0.2}$ in reasonable agreement with the results of numerical simulations (Ruffert \& Janka 1999). We construct accreting disks i.e. disks with a cusp in the equatorial plane where the total gravitational + centrifugal force is zero. If $a=0$, we model the black hole with the Paczynski-Wiita potential while for a Kerr black hole we use the Novikov potential (Artemova et al. 1996)

$\Phi_{\mathrm{BH}}(r)=-\frac{G M_{\mathrm{BH}}}{(\beta-1) r_{\mathrm{h}}}\left[\left(\frac{r}{r-r_{\mathrm{h}}}\right)^{\beta-1}-1\right]$,

with

$r_{\mathrm{h}}=\left(1+\sqrt{1-a^{2}}\right) r_{\mathrm{g}} \quad$ and $\quad \beta=\frac{r_{\mathrm{ms}}}{r_{\mathrm{h}}}-1$,

where $r_{\mathrm{g}}=G M_{\mathrm{BH}} / c^{2}$ is the gravitational radius, $r_{\mathrm{h}}$ is the horizon radius and $r_{\mathrm{ms}}$ the radius of the last stable orbit. We have considered three cases: $M_{\mathrm{BH}}=2.5 M_{\odot}$, $M_{\mathrm{D}}=0.3 M_{\odot}$ with $a=0$ (case 1$)$ and $a=0.8$ (case 2); $M_{\mathrm{BH}}=5 M_{\odot}, M_{\mathrm{D}}=0.5 M_{\odot}$ with $a=0.4$ (case 3 ). Cases 1 and 2 are representative of NS+NS mergers while case 3 corresponds to a $\mathrm{BH}+\mathrm{NS}$ merger. The obtained disk structures are represented in Fig. 1. Compared to case 1, the increase of $a$ in case 2 leads to a denser $\left(\rho_{\max }=4.2 \times\right.$ $10^{12}$ instead of $3.3 \times 10^{11} \mathrm{~g} \mathrm{~cm}^{-3}$ ) and more compact disk (the radius at maximum density $r_{\max }$ and the external 
radius $r_{\text {ext }}$ are 58 and $279 \mathrm{~km}$ in case 1,25 and $117 \mathrm{~km}$ in case 2 ). In case 3 , the maximum density is $\rho_{\max }=$ $4.3 \times 10^{11} \mathrm{~g} \mathrm{~cm}^{-3}$ at $r_{\max }=80.4 \mathrm{~km}$ and the external radius is $r_{\text {ext }}=261 \mathrm{~km}$. We have computed the optical thickness of these disks to electron neutrinos in the vertical direction

$\tau_{\nu_{\mathrm{e}}}=\int_{-\infty}^{+\infty} \kappa_{\nu_{\mathrm{e}}} \rho \mathrm{d} z$

where

$\kappa_{\nu_{\mathrm{e}}}=3.8 \times 10^{-19}\left(\frac{T_{\nu}}{1 \mathrm{MeV}}\right)^{2} \mathrm{~cm}^{2} \mathrm{~g}^{-1}$

is the neutrino opacity (Duncan et al. 1986). The radial extension of the optically thick region has been represented in Fig. 1 for two temperatures, $T_{\nu}=2$ and $3 \mathrm{MeV}$. The optical thickness becomes larger than unity at $3-5 r_{\mathrm{h}}$ and the disk remains opaque out to $r_{\text {out }} \gtrsim 10 r_{\mathrm{h}}$.

When the disk is optically thick we use the following expression for the neutrinosphere temperature

$T_{\nu}(r)=T_{*}\left(\frac{r_{*}}{r}\right)^{3 / 4}\left(\frac{1-\sqrt{\frac{r_{\mathrm{in}}}{r}}}{1-\sqrt{\frac{r_{\mathrm{in}}}{r_{*}}}}\right)^{1 / 4}$

where $r_{\text {in }}$ is the disk internal radius and $T_{*}$ is the temperature at a reference radius $r_{*}$ (we take below $r_{*}=4 r_{\mathrm{h}}$ ). This kind of behavior is expected in the case of a geometrically thin disk but is certainly a very rough approximation in the case of a thick disk.

\subsection{Collapsars}

In collapsars the accretion flow toward the black hole has been studied in detail by Popham et al. (1999). The inner disk is fed by material from the collapsing stellar enveloppe. It is less dense than in the merger case (typical densities are $10^{8} \mathrm{~g} / \mathrm{cm}^{3}$ ) and is optically thin (MacFadyen \& Woosley 1999). We follow Popham et al. (1999) to obtain an analytical expression for the temperature from the balance between the dissipated energy and neutrino losses

$\dot{q}_{\mathrm{eN}}=Q T^{6}=\frac{9}{4} \nu \Omega_{\mathrm{K}}^{2}$

where $\dot{q}_{\mathrm{eN}}$ is the cooling rate per unit mass due to the emission of neutrinos by nucleons $\left(Q=1.4 \times 10^{18}\right.$ with $T$ in $\mathrm{MeV}) ; \dot{q}_{\mathrm{eN}}$ is the dominant cooling contribution as long as the disk temperature does not exceed about $10 \mathrm{MeV}$. We adopt an $\alpha$-prescription for the disk viscosity

$\nu=\alpha H v_{\mathrm{s}}$

where $H=\frac{v_{\mathrm{s}}}{\Omega_{\mathrm{K}}}$ is the disk half thickness and $v_{\mathrm{s}}$ is the sound velocity. If the perfect gas contribution dominates in the disk then, $v_{\mathrm{s}}^{2} \sim \frac{\mathcal{R} T}{\mu}$ ( $\mu$ being the average molecular weight) and the disk temperature is given by

$T_{\mathrm{d}}(r) \simeq 2 \mu_{\mathrm{BH}}^{-0.2}\left(\frac{\alpha}{0.01}\right)^{0.2}\left(\frac{r}{r_{*}}\right)^{-0.3} \mathrm{MeV}$.
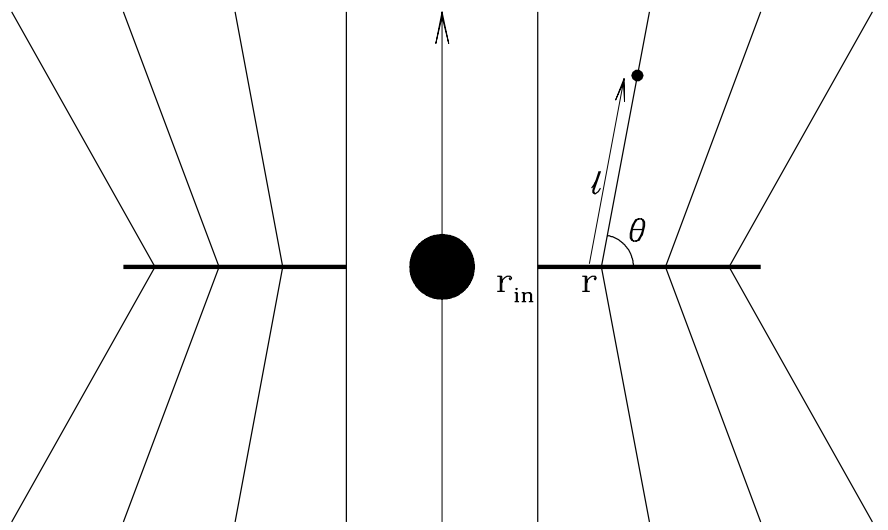

Fig. 2. Schematic view of the system geometry. The disk is assumed to be geometrically thin and the magnetic field lines make an angle $\theta$ with the disk. The outflow follow the field lines and the position of a fluid element along a line anchored at a radial distance $r$ from the black hole is represented by $y=\ell / r$.

\section{Dynamics of the wind from the disk to the sonic point}

\subsection{Wind equations}

To study the dynamics of the wind we make a number of simplifying assumptions. We first suppose that the inner disk is geometrically thin which is certainly wrong in the merger case and probably a poor approximation in the collapsar scenario. Wind material is guided along magnetic field lines for which we adopt the simplest possible geometry: close to the disk the field is poloidal, made of straight lines making an angle $\theta(r)$ with the plane of the disk ( $r$ being the distance from the foot of the line to the disk axis; see Fig. 2). Since we limit our study to the part of the flow below the sonic point (which is sufficient to obtain the mass loss rate) we use non relativistic equations $(v / c \ll 1)$ but we adopt a Paczynski-Wiita potential for the black hole. We also assume that the wind has reached a stationary regime. Clearly, a realistic description would imply a thick disc, a complicated field geometry and a time-dependent wind dynamics but we believe that the toy model presented in this paper remains able to identify the main physical processes which affects the baryonic pollution of the wind.

We write the three flow equations in a frame corotating with the foot of the line:

- Conservation of mass:

$$
\rho v s(y)=\dot{m},
$$

- Euler equation:

$$
v \frac{\mathrm{d} v}{\mathrm{~d} y}=\gamma(y) r-\frac{1}{\rho} \frac{\mathrm{d} P}{\mathrm{~d} y},
$$

- Energy equation:

$$
v \frac{\mathrm{d} e}{\mathrm{~d} y}=\dot{q}(y) r+v \frac{P}{\rho^{2}} \frac{\mathrm{d} \rho}{\mathrm{d} y},
$$


where $y=\ell / r, \ell$ being the distance along the field line $(\ell=0$ in the plane of the disk); $e$ is the specific internal energy, $\gamma(y)$ and $\dot{q}(y)$ are respectively the total acceleration (gravitational + centrifugal) and the power deposited per unit mass in wind material due to neutrino heating (and cooling), viscous or ohmic dissipation and magnetic reconnection. Because the field and stream lines are coincident the function $s(y)$ can be simply related to the field geometry. We get

$s(y)=1+a y+b y^{2}$

with

$a=\cos \theta-\sin \theta \frac{\mathrm{d} \theta}{\mathrm{d} \log r}$

and

$b=-\cos \theta \sin \theta \frac{\mathrm{d} \theta}{\mathrm{d} \log r}$.

Finally, $\dot{m}$ is the mass loss rate per unit surface of the disk. The acceleration $\gamma(y)$ is derived from the potential

$\gamma(y) r=-\frac{\mathrm{d} \Phi}{\mathrm{d} y}, \quad \Phi(y)=\Phi_{\mathrm{BH}}(y)+\Phi_{\mathrm{C}}(y)$,

where

$$
\begin{aligned}
\Phi_{\mathrm{BH}}(y) & =-\frac{G M_{\mathrm{BH}}}{r \sqrt{y^{2}+2 y \cos \theta+1}-r_{\mathrm{h}}} \\
& =-\frac{c^{2}}{2}\left[\frac{1}{x \sqrt{y^{2}+2 y \cos \theta+1}-1}\right]
\end{aligned}
$$

is the black hole potential $\left(x=r / r_{\mathrm{h}}\right)$ and

$$
\begin{aligned}
\Phi_{\mathrm{C}}(y) & =-\frac{1}{2} \Omega^{2}(r) r^{2}(1+y \cos \theta)^{2} \\
& =-\frac{c^{2}}{4}\left[\frac{x}{(x-1)^{2}}(1+y \cos \theta)^{2}\right]
\end{aligned}
$$

is the centrifugal potential. There is a critical angle $\theta_{\mathrm{cr}}$ below which the acceleration is always positive so that matter (even at zero temperature) can escape freely from the disk without being confined in a potential well. In newtonian gravity $\theta_{\mathrm{cr}}=60^{\circ}$ (Blandford \& Payne 1982) but $\theta_{\text {cr }}$ slowly decreases when the Paczynski-Wiita potential is used, from about $63^{\circ}$ at $x=3$ to $60^{\circ}$ at large radial distances.

\subsection{Equation of state}

Our equation of state includes nucleons, relativistic electrons and positrons, and photons. Following Bethe et al. (1980) and Bethe (1993) the contribution of relativistic particles is given by

$$
\begin{aligned}
P_{\mathrm{r}} & =\frac{(k T)^{4}}{12(\hbar c)^{3}}\left(\frac{11 \pi^{2}}{15}+2 \eta^{2}+\frac{\eta^{4}}{\pi^{2}}\right) \\
& =\left(1.26+0.35 \eta^{2}+0.017 \eta^{4}\right) 10^{26} T_{\mathrm{MeV}}^{4} \text { dyne } \mathrm{cm}^{-2}
\end{aligned}
$$

where $\eta=\mu_{\mathrm{e}} / k T, \mu_{\mathrm{e}}$ being the electron chemical potential. Nucleons behave as an ideal gas of pressure

$P_{\mathrm{N}}=\frac{\rho}{m_{\mathrm{N}}} k T$

where $m_{\mathrm{N}}$ is the nucleon mass and the density is obtained from

$$
\begin{aligned}
\rho & =\frac{m_{\mathrm{N}}}{3}\left(\frac{k T}{\hbar c}\right)^{3} Y_{\mathrm{e}}^{-1}\left(\eta+\frac{\eta^{3}}{\pi^{2}}\right) \\
& =7.2 \times 10^{7} T_{\mathrm{MeV}}^{3} Y_{\mathrm{e}}^{-1}\left(\eta+\frac{\eta^{3}}{\pi^{2}}\right) \mathrm{g} \mathrm{cm}^{-3} .
\end{aligned}
$$

The number of electrons per nucleon $Y_{\mathrm{e}}$ should be computed from the rates of neutrino capture and emission by nucleons. In practice, we do not perform this calculation and simply adopt a constant $Y_{\mathrm{e}}=0.5$. Finally, the specific internal energy is

$e=\frac{\left(3 P_{\mathrm{r}}+3 / 2 P_{\mathrm{N}}\right)}{\rho}$

so that the three thermodynamic quantities $P, \rho$ and $e$ that appear in the flow equations can be expressed in terms of $T$ and $\eta$.

\subsection{The sonic point}

To solve the flow Eqs. (9)-(11) we first derive Eq. (9) and express the thermodynamic variables $\rho, P$ and $e$ as functions of $T$ and $\eta$ to obtain a linear system for $\log v$, $\log T$ and $\log \eta$

$$
\begin{aligned}
& \frac{\mathrm{d} \log v}{\mathrm{~d} y}+3 \frac{\mathrm{d} \log T}{\mathrm{~d} y}+A(\eta) \frac{\mathrm{d} \log \eta}{\mathrm{d} y}=-\frac{\mathrm{d} \log s(y)}{\mathrm{d} y}, \\
& v^{2} \frac{\mathrm{d} \log v}{\mathrm{~d} y}+4 \frac{P}{\rho} \frac{\mathrm{d} \log T}{\mathrm{~d} y}+B(\eta) \frac{\mathrm{d} \log \eta}{\mathrm{d} y}=\gamma(y) r, \\
& v\left[e-3 \frac{P}{\rho}\right] \frac{\mathrm{d} \log T}{\mathrm{~d} y}+v\left[C(\eta) e-A(\eta) \frac{P}{\rho}\right] \frac{\mathrm{d} \log \eta}{\mathrm{d} y}=\dot{q}(y) r,
\end{aligned}
$$

where we have used the equation of state to get

$$
\begin{aligned}
& \left.\frac{\partial \log \rho}{\partial \log T}\right|_{\eta}=3,\left.\frac{\partial \log \rho}{\partial \log \eta}\right|_{T}=A(\eta), \\
& \left.\frac{\partial \log P}{\partial \log T}\right|_{\eta}=4,\left.\frac{\partial \log P}{\partial \log \eta}\right|_{T}=B(\eta), \\
& \left.\frac{\partial \log e}{\partial \log T}\right|_{\eta}=1,\left.\frac{\partial \log e}{\partial \log \eta}\right|_{T}=C(\eta) .
\end{aligned}
$$

The three derivatives of $v, T$ and $\eta$ can then be written as

$$
\begin{aligned}
& \frac{\mathrm{d} \log v}{\mathrm{~d} y}=\frac{F_{1}(y, v, T, \eta)}{\Delta}, \\
& \frac{\mathrm{d} \log T}{\mathrm{~d} y}=\frac{F_{2}(y, v, T, \eta)}{\Delta}, \\
& \frac{\mathrm{d} \log \eta}{\mathrm{d} y}=\frac{F_{3}(y, v, T, \eta)}{\Delta},
\end{aligned}
$$


where $\Delta(v, T, \eta)$ is the determinant of the system. It is equal to zero at the sonic point which gives

$v^{2}=v_{\mathrm{s}}^{2}=\left[\frac{(B-4 C)-(\tilde{\gamma}-1)(3 B-4 A)}{(A-3 C)}\right] \frac{P}{\rho}$

where $\tilde{\gamma}-1=P / \rho e$. If the equation of state is dominated by the contribution of relativistic (resp. non relativistic) particles $\tilde{\gamma}-1=1 / 3($ resp. $2 / 3)$ and $v_{\mathrm{s}}^{2}=\frac{4}{3}\left(\operatorname{resp} . \frac{5}{3}\right) \frac{P}{\rho}$.

Since the three functions $v, T$ and $\eta$ must remain regular everywhere in the wind the numerators in Eq. (24) must be zero at the sonic point

$F_{1}\left(y_{\mathrm{s}}, v_{\mathrm{s}}, T_{\mathrm{s}}, \eta_{\mathrm{s}}\right)=F_{2}\left(y_{\mathrm{s}}, v_{\mathrm{s}}, T_{\mathrm{s}}, \eta_{\mathrm{s}}\right)=F_{3}\left(y_{\mathrm{s}}, v_{\mathrm{s}}, T_{\mathrm{s}}, \eta_{\mathrm{s}}\right)=0$

which yields a unique relation among the parameters at the sonic point

$\left.v_{\mathrm{s}}^{2} \frac{\mathrm{d} \log s(y)}{\mathrm{d} y}\right|_{y_{\mathrm{s}}}+\gamma\left(y_{\mathrm{s}}\right) r-\frac{\dot{q}\left(y_{\mathrm{s}}\right) r}{v_{\mathrm{s}}}\left(\frac{P}{\rho e} \frac{4 A-3 B}{A-3 C}\right)_{T_{\mathrm{s}}, \eta_{\mathrm{s}}}=0$

the parenthesis being equal to $1 / 3$ (resp. 2/3) for relativistic (resp. non relativistic) particles. Since (i) the last term in Eq. (27) is smaller than the two others in most cases of interest and (ii) the derivative of $s(y)$ is positive (if the inclination angle $\theta(r)$ of the field lines decreases with increasing distance to the axis) it can be seen that the sonic point is located in the region where $\gamma(y)$ is negative, i.e. below $y_{1}$ where $\gamma\left(y_{1}\right)=0$. It appears in practice that $y_{\mathrm{s}}$ is very close to $y_{1}$ (except naturally for $\theta=90^{\circ}$ for which $\left.y_{1} \rightarrow \infty\right)$. The difference is typically less than $1 \%$ even for $\theta=89^{\circ}$.

\subsection{Heating and cooling sources}

Several sources can contribute to the injection of energy in wind material: viscous or ohmic dissipation, magnetic reconnection or neutrino processes (capture on free nucleons, scattering on electrons and positrons or neutrino-antineutrino annihilation). Cooling occurs through neutrino emission by nucleons and annihilation of electron-positron pairs. A detailed description of all these processes is beyond the scope of this paper and we have rather considered two limiting cases in a very simplified way.

When the disk is optically thin to neutrinos we adopt a uniform heating (per unit mass) $\dot{q}_{\mathrm{h}}$ along the field line and limit the cooling to neutrino emission by nucleons i.e.

$\dot{q}=\dot{q}_{\mathrm{h}}-Q T^{6}$.

Although this is not strictly satisfied in realistic models (Popham et al. 1999; MacFadyen \& Woosley 1999), we require that $\dot{q}=0$ in the plane of the disk which fixes $\dot{q}_{\mathrm{h}}$

$\dot{q}_{\mathrm{h}}=Q T_{0}^{6}$

where $T_{0}$ is the disk temperature at the foot of the line given by Eq. (8).
In the case of an optically thick disk we make the extreme assumption that all the heating which is not due to neutrino processes takes place below the neutrinosphere. The power $\dot{q}$ injected into the wind is then restricted to the neutrino contributions

$\dot{q}=\dot{q}_{\nu}=\dot{q}_{\nu \mathrm{N}}+\dot{q}_{\nu \mathrm{e}}+\dot{q}_{\nu \bar{\nu}}-\dot{q}_{\mathrm{eN}}-\dot{q}_{\mathrm{e}^{+} \mathrm{e}^{-}}$

where the different terms in Eq. (30) respectively correspond to neutrino capture on free nucleons, scattering on electrons and positrons, neutrino-antineutrinoannihilation (heating) neutrino emission by nucleons and annihilation of electron-positron pairs (cooling). With our assumption that $Y_{\mathrm{e}}=Y_{\mathrm{p}}=Y_{\mathrm{n}}=0.5$ the electron neutrino and antineutrino temperatures are identical and the heating by capture on free nucleons takes the simple form (Qian \& Woosley 1996)

$$
\begin{aligned}
\dot{q}_{\nu \mathrm{N}} & =Q \int_{\text {disk }}\left[I_{\nu_{\mathrm{e}}} \frac{\left\langle\epsilon_{\nu_{\mathrm{e}}}^{3}\right\rangle}{\left\langle\epsilon_{\nu_{\mathrm{e}}}\right\rangle}+I_{\bar{\nu}_{\mathrm{e}}} \frac{\left\langle\epsilon_{\bar{\nu}_{\mathrm{e}}}^{3}\right\rangle}{\left\langle\epsilon_{\bar{\nu}_{\mathrm{e}}}\right\rangle}\right] \mathrm{d} \Omega \\
& =1.4 \times 10^{18} \int_{\text {disk }} T_{\nu}^{6} \frac{\mathrm{d} \Omega}{4 \pi} \mathrm{erg} \mathrm{g}^{-1} \mathrm{~s}^{-1}
\end{aligned}
$$

where the temperature is in $\mathrm{MeV}, I_{\nu_{\mathrm{e}}}=I_{\bar{\nu}_{\mathrm{e}}}=\frac{7}{16} \frac{\sigma T_{\nu}^{4}}{\pi}$ is the neutrino (antineutrino) intensity and $\left\langle\epsilon_{\nu_{\mathrm{e}}}^{n}\right\rangle=\left\langle\epsilon_{\bar{\nu}_{\mathrm{e}}}^{n}\right\rangle^{\pi}$ are the $n$th energy moments of the neutrino Fermi distribution. The integral is performed over the disk surface and $\mathrm{d} \Omega$ is the solid angle of a surface element as seen from a point of coordinate $y$ on the field line. All relativistic effects on the neutrinos (bending of trajectories, gravitational and Doppler shifts) have been neglected even if they can lead to appreciable corrections in the results (Jaroszynski 1993). The cooling by the reverse reactions (neutrino emission by nucleons) is given by

$\dot{q}_{\mathrm{eN}}=1.4 \times 10^{18} \mathrm{~T}^{6} \mathrm{erg} \mathrm{g}^{-1} \mathrm{~s}^{-1}$

where $T$ is the local temperature in the wind. Pauli blocking effects for electrons have been neglected since $\eta \sim 0.1$ everywhere in our wind solutions except in the vicinity of the disk. To compute the heating rate due to neutrino scattering on relativistic electrons and positrons we use the expression given by Herant et al. (1994) adapted to the disk geometry

$\dot{q}_{\nu \mathrm{e}}=3.6 \times 10^{24} \frac{T^{4}}{\rho} \int_{\text {disk }}\left(T_{\nu}-T\right) T_{\nu}^{4} \mathrm{~d} \Omega \operatorname{erg~g}^{-1} \mathrm{~s}^{-1}$.

The heating rate by neutrino-antineutrino annihilation is obtained from a double integral over the disk surface

$$
\begin{aligned}
& \dot{q}_{\nu \bar{\nu}}=\frac{1}{\rho}\left\{Q_{1} \int_{\text {disk }} \mathrm{d} \Omega I_{\nu_{\mathrm{e}}} \int_{\text {disk }} \mathrm{d} \Omega^{\prime} I_{\bar{\nu}_{\mathrm{e}}}^{\prime}\left[\frac{\left\langle\epsilon_{\nu_{\mathrm{e}}}^{2}\right\rangle}{\left\langle\epsilon_{\nu_{\mathrm{e}}}\right\rangle}+\frac{\left\langle\epsilon_{\bar{\nu}_{\mathrm{e}}}^{2}\right\rangle^{\prime}}{\left\langle\epsilon_{\bar{\nu}_{\mathrm{e}}}\right\rangle^{\prime}}\right](1-\cos \alpha)^{2}\right. \\
& \left.+Q_{2} \int_{\text {disk }} \mathrm{d} \Omega I_{\nu_{\mathrm{e}}} \int_{\text {disk }} \mathrm{d} \Omega^{\prime} I_{\bar{\nu}_{\mathrm{e}}}^{\prime} \frac{\left\langle\epsilon_{\nu_{\mathrm{e}}}\right\rangle+\left\langle\epsilon_{\bar{\nu}_{\mathrm{e}}}\right\rangle^{\prime}}{\left\langle\epsilon_{\nu_{\mathrm{e}}}\right\rangle\left\langle\epsilon_{\bar{\nu}_{\mathrm{e}}}\right\rangle^{\prime}}(1-\cos \alpha)\right\}
\end{aligned}
$$

where $Q_{1}$ and $Q_{2}$ are two constants given in Ruffert et al. (1997). The prime quantities correspond to a surface 

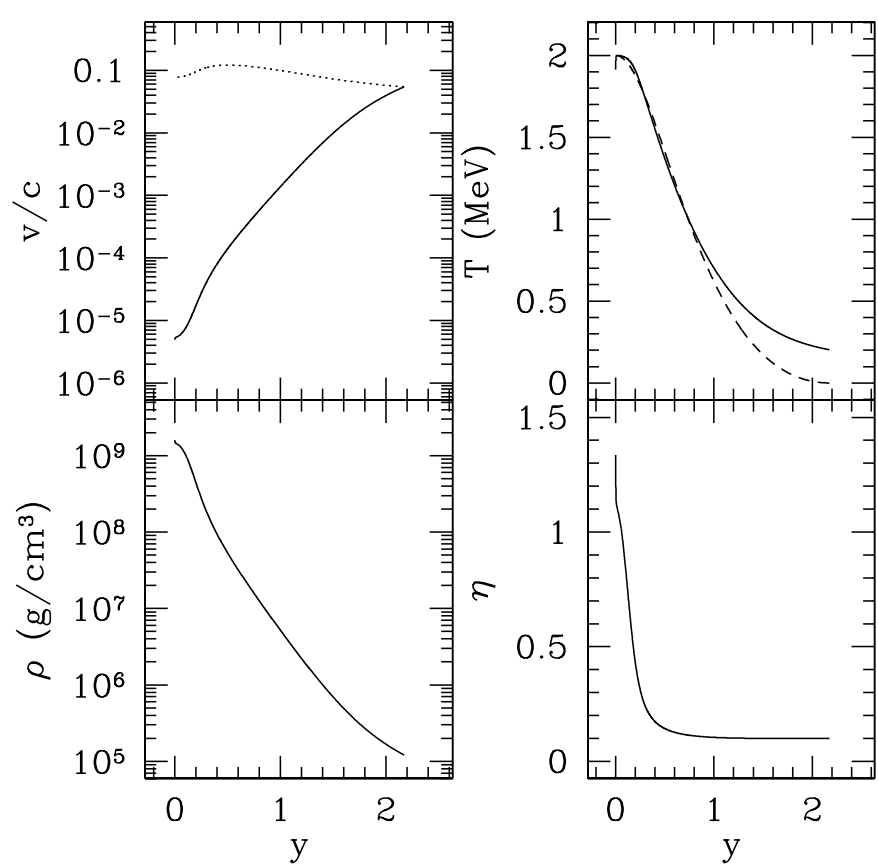

Fig. 3. Wind solution for an optically thin disk with uniform heating. The field line is anchored at $r_{*}=4 r_{\mathrm{h}}$ and makes an angle $\theta=85^{\circ}$ with the disk. The temperature at the foot of the line is $T_{\mathrm{d}}=2 \mathrm{MeV}$. In the velocity plot the dotted line shows the local sound velocity. The sonic point is located at $y_{\mathrm{s}}=$ 2.174. The dashed line in the temperature plot corresponds to the approximate analytical solution (Eq. (47)). The electron degeneracy parameter $\eta=\mu_{\mathrm{e}} / k T$ is close to 0.1 except at the vicinity of the disk.

element $\mathrm{d} S^{\prime}$ whose neutrinos interact with those emitted by a surface element $\mathrm{d} S, \alpha$ being the interaction angle. When the neutrino intensities and average energies are expressed as a function of the neutrinosphere temperature $T_{\nu}$ Eq. (34) becomes

$$
\begin{aligned}
\dot{q}_{\nu \bar{\nu}}= & \frac{1}{\rho}\left\{1.6 \times 10^{22} \int_{\text {disk }} \mathrm{d} \Omega T_{\nu}^{4} \int_{\text {disk }} \mathrm{d} \Omega^{\prime} T_{\nu}^{\prime 4}\left(T_{\nu}+T_{\nu}^{\prime}\right)(1-\cos \alpha)^{2}\right. \\
& \left.+6.7 \times 10^{20} \int_{\text {disk }} \mathrm{d} \Omega T_{\nu}^{4} \int_{\text {disk }} \mathrm{d} \Omega^{\prime} T_{\nu}^{\prime} \frac{T_{\nu}+T_{\nu}^{\prime}}{T_{\nu} T_{\nu}^{\prime}}(1-\cos \alpha)\right\} \\
& \operatorname{erg~g}^{-1} \mathrm{~s}^{-1} .
\end{aligned}
$$

Finally, the cooling rate from the annihilation of electronpositron pairs is given by (Herant et al. 1994)

$q_{\mathrm{e}^{+} \mathrm{e}^{-}}=1.5 \times 10^{25} \frac{T^{9}}{\rho} \operatorname{erg~g}^{-1} \mathrm{~s}^{-1}$.

\section{The mass loss rate}

\subsection{Numerical solution for an optically thin disk}

We first solve the flow equations for an optically thin disk and in the case of uniform heating, i.e. with $\dot{q}$ given by Eq. (28). We obtain the mass loss rate $\dot{m}$ in a classical way by integrating inward from the sonic point down to the disk surface. We fix trial values of $T_{\mathrm{s}}$ and $\eta_{\mathrm{s}}$ at the sonic point from which we get $v=v_{\mathrm{s}}$ from Eq. (25) and the position $y_{\mathrm{s}}$ from Eq. (27). To start the integration we need the derivatives of $v, T$ and $\eta$ at $y=y_{\mathrm{s}}$. These derivatives cannot be directly calculated from Eq. (24) because $F_{1}=F_{2}=F_{3}=\Delta=0$ at the sonic point. We instead use l'Hôpital's rule which allows us to write three algebraic, second-order equations for the three derivatives and we solve these equations with a Newton-Raphson technique. Once $v, T, \eta$ and their derivatives have been determined at the sonic point the inward integration can be started. In agreement with the results of Duncan et al. (1986) for neutrino-driven winds in proto-neutron stars we observe that at some position $y=y_{*}$ the velocity begins to fall off rapidly while the temperature reaches a maximum $T_{\max } \leq T_{\mathrm{d}}(r)\left(T_{\mathrm{d}}(r)\right.$ being the disk temperature at radius $r)$. We then adjust the values of $T_{\mathrm{s}}$ and $\eta_{\mathrm{s}}$ with the requirement that $y_{*}$ should be as close as possible to 0 and $T_{\max }$ to $T_{\mathrm{d}}(r)$.

We first constructed a reference model where we follow the wind along a field line attached at $r=r_{*}=4 r_{\mathrm{h}}$. The disk temperature at $r=r_{*}$ is $T_{\mathrm{d}}=2 \mathrm{MeV}$. The line makes an angle $\theta=85^{\circ}$ with the disk and the derivative $\frac{\mathrm{d} \theta}{\mathrm{d} r}=0$. The mass of the black hole is $M_{\mathrm{BH}}=2.5 M_{\odot}$. The results for this reference model are shown in Fig. 3. The sonic point is located at $y_{\mathrm{s}}=2.174$ slightly below $y_{1}=2.182$ where $\gamma=0$. The temperature and density at the sonic point are $T_{\mathrm{s}}=0.203 \mathrm{MeV}$ and $\rho_{\mathrm{s}}=1.2 \times 10^{5} \mathrm{~g} \mathrm{~cm}^{-3}$ which correspond to a sound velocity $v_{\mathrm{s}} / c=5.44 \times 10^{-2}$ or $v_{\mathrm{s}}=16300 \mathrm{~km} \mathrm{~s}^{-1}$ and a mass loss rate $\dot{m}=2.3 \times 10^{14} \mathrm{~g} \mathrm{~cm}^{-2} \mathrm{~s}^{-1}$. The degeneracy parameter $\eta$ remains practically constant $(\eta \simeq 0.1)$ from $y=0.5$ to the sonic point, a property which will be used to construct the analytical solutions in Sect. 4.3 below. Neutrino cooling is efficient close to the disk, up to $y \lesssim 0.5$. We have tested the effect of a different field line geometry with $\theta^{\prime}=\frac{\mathrm{d} \theta}{\mathrm{d} r} \neq 0$. We considered two cases, $\theta^{\prime}=-2^{\circ} / r_{\mathrm{h}}$ and $-5^{\circ} / r_{\mathrm{h}}$, for which we respectively obtain $\dot{m}=2.5$ and $2.8 \times 10^{14} \mathrm{~g} \mathrm{~cm}^{-2} \mathrm{~s}^{-1}$.

We then varied the mass of the black hole $M_{\mathrm{BH}}$, the disk temperature $T_{\mathrm{d}}$, the inclination angle $\theta$ and the position $r$ of the foot of the line to see how these parameters affect the mass loss rate. The results are shown in Fig. 4 where we have plotted $\dot{m}$ when $M_{\mathrm{BH}}, T_{\mathrm{d}}, \theta$ and $x=r / r_{\mathrm{h}}$ are varied separately while the other three quantities are maintained at fixed values (choosen to be those of the reference model: $M_{\mathrm{BH}}=2.5 M_{\odot}, T_{\mathrm{d}}=2 \mathrm{MeV}, \theta=85^{\circ}$ and $x=4)$. The mass loss rate appears to be nearly proportional to the mass of the black hole (Fig. 4a). The dependence of $\dot{m}$ on the temperature $T_{\mathrm{d}}$ at the foot of the field line is much more spectacular since we get $\dot{m} \propto T_{\mathrm{d}}^{10}$ (Fig. 4b) in agreement with the results for neutrino-driven winds in neutron stars (Duncan et al. 1986). The mass loss rate also sharply increases when the angle between the field line and the disk is reduced (Fig. 4c). Below $\theta \simeq 77^{\circ}$ 

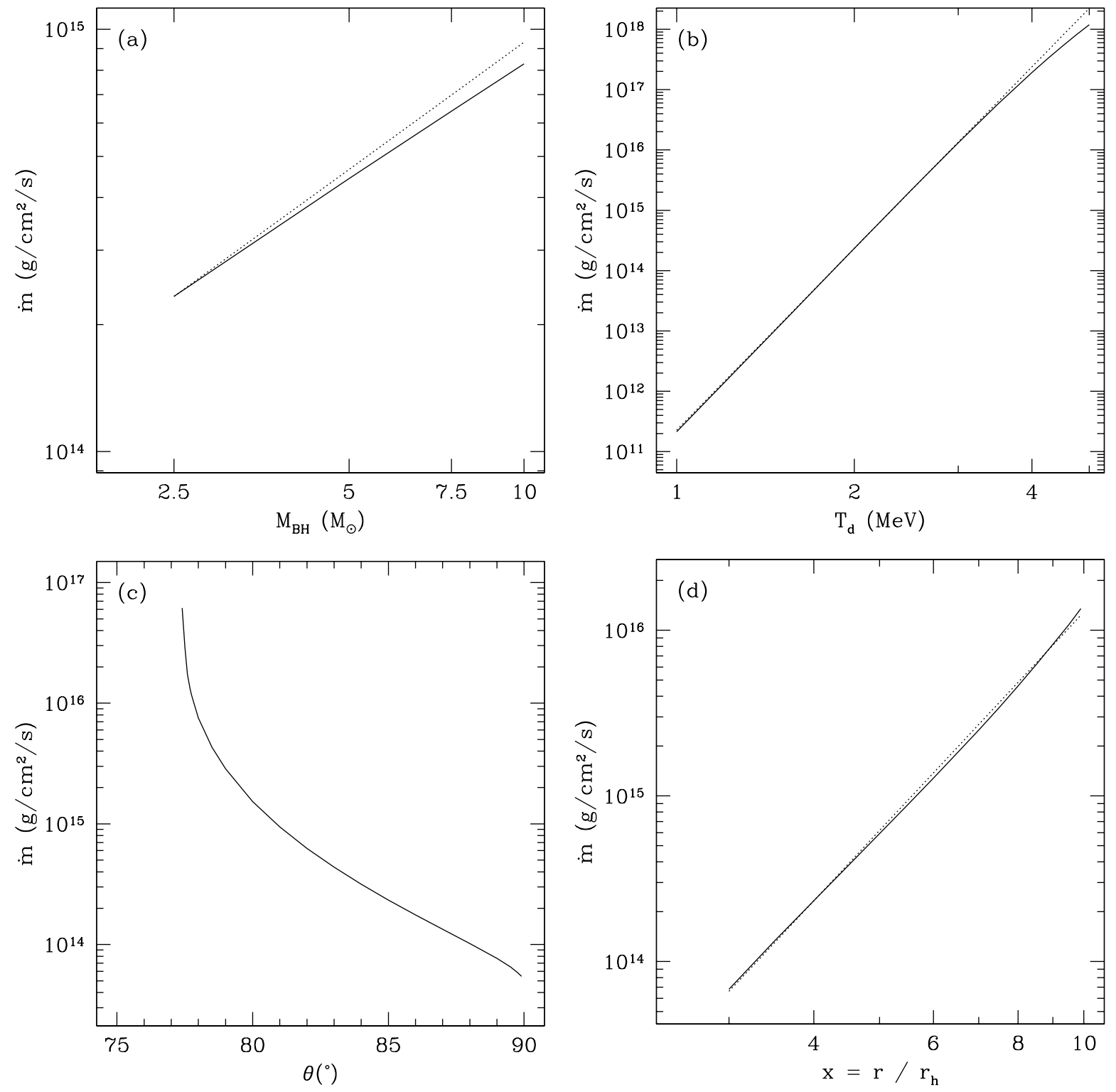

Fig. 4. Mass loss rate per unit surface for an optically thin disk with uniform heating as a function of a) black hole mass $M_{\mathrm{BH}}$, b) disk temperature at the foot of the line $T_{\mathrm{d}}, \mathbf{c}$ ) inclination angle $\theta$ and $\mathbf{d}$ ) radial distance to the black hole. When one quantity is varied the three others are maintained at their reference values: $M_{\mathrm{BH}}=2.5 M_{\odot}, T_{\mathrm{d}}=2 \mathrm{MeV}, \theta=85^{\circ}$ and $r=4 r_{\mathrm{h}}$. The dotted lines in $\mathbf{a}), \mathbf{b}$ ) and $\mathbf{d}$ ) have respective slopes 1,10 and 4.4 .

it becomes more and more difficult to construct wind solutions with the required accuracy. This can be related to the value of the Bernouilli function

$\mathcal{B}=\frac{1}{2} v^{2}+h+\Phi-\Phi_{1}$

( $h$ being the specific enthalpy and $\Phi_{1}$ the potential at $y=$ $\left.y_{1}\right)$ which is positive in the plane of the disk for $\theta \leq 77^{\circ}$. The initial thermal energy is then sufficient to allow the gas to escape even in the absence of additional heating. Finally, the mass loss rate approximately increases as $r^{4.4}$ (Fig. 4d) when the potential well becomes shallower at larger radial distances.
As long as the Bernouilli function is not too close to zero the mass, temperature and geometrical dependence can be separated in $\dot{m}$ to yield the general expression

$\dot{m}(x) \approx 2.3 \times 10^{14} \mu_{\mathrm{BH}}\left[\frac{T_{\mathrm{d}}(x)}{2 \mathrm{MeV}}\right]^{10} f[x, \theta(x)] \mathrm{g} \mathrm{cm}^{-2} \mathrm{~s}^{-1}$,

where $f$ is a geometrical function which satisfies $f\left(4,85^{\circ}\right)=1$. The mass loss rate has been represented in Fig. 5 as a function of $x$ for $\mu_{\mathrm{BH}}=1, T_{\mathrm{d}}(x)$ given by Eq. (8) and different field geometries: constant inclination angles $\theta=80,85$ and $89^{\circ}$ or $\theta$ linearly decreasing from $90^{\circ}$ at $x=3$, i.e. $\theta=90^{\circ}-\lambda(x-3)$ (with $\lambda=0.5,1.0,1.5$ or $2^{\circ}$ ). At constant $\theta$ the mass loss rate varies rather slowly 


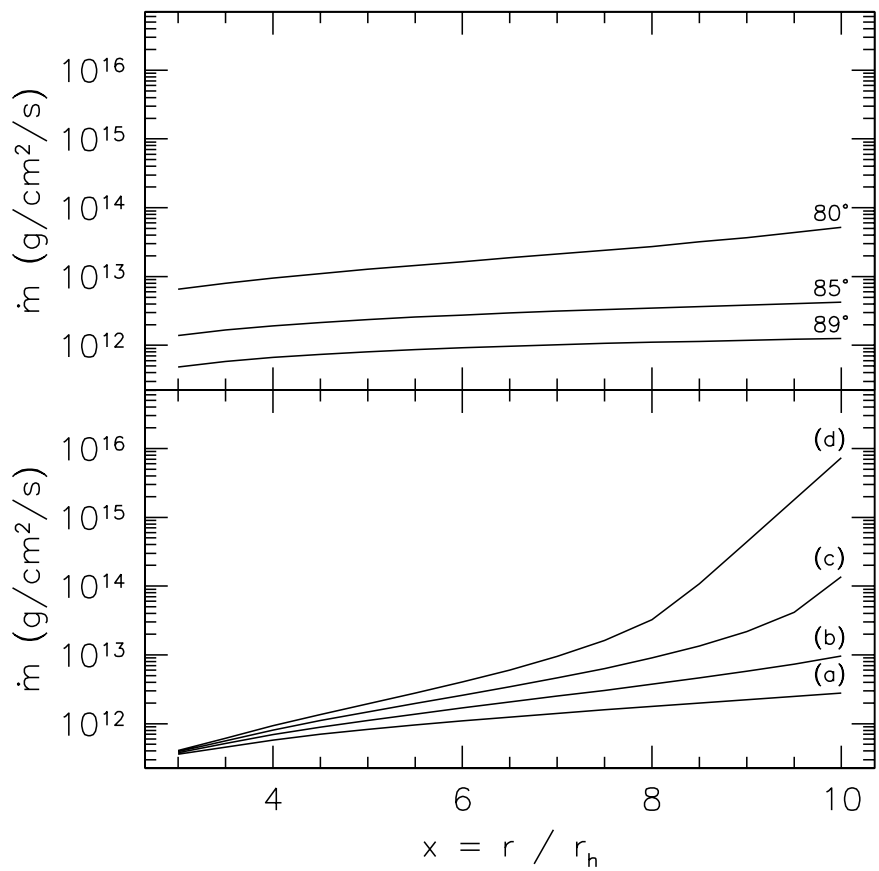

Fig. 5. Mass loss rate per unit surface as a function of $x=r / r_{\mathrm{h}}$ for an optically thin disk with uniform heating. Upper panel: field lines with constant inclination angles $\theta=80,85$ and $89^{\circ}$; lower panel: field lines with decreasing inclination angle $\theta=$ $90^{\circ}-\lambda(x-3)$ with $\lambda=0.5,1,1.5$ and $2^{\circ}$ (curves labelled a) to d)).

with $x$ while for $\lambda=1.5$ or $2^{\circ}$ it rapidly increases at large radial distances where $\theta \leq 80^{\circ}$.

\subsection{Numerical solution for an optically thick disk}

If the disk is optically thick the heating and cooling sources due to neutrinos are specified by Eqs. (31)-(36). Again, wind solutions are found by inward integration from the sonic point down to the disk. A reference model is constructed with the same black hole mass and field geometry $\left(M_{\mathrm{BH}}=2.5 M_{\odot}\right.$ and field line attached at $r=r_{*}=4 r_{\mathrm{h}}$ and making an angle $\theta=85^{\circ}$ with the disk). The disk is supposed to be optically thick to neutrinos from $r_{\text {in }}=3 r_{\mathrm{h}}$ to $r_{\text {out }}=10 r_{\mathrm{h}}$. The temperature of the neutrinosphere in this reference model is $T_{\nu}=2 \mathrm{MeV}$ and does not vary with radius which allows a simple calculation of the geometric integrals appearing in the neutrino heating terms. The results for this reference model are shown in Fig. 6. The sonic point is located at $y_{\mathrm{s}}=2.175$. The temperature and density at the sonic point are $T_{\mathrm{s}}=$ $0.132 \mathrm{MeV}$ and $\rho_{\mathrm{s}}=3.23 \times 10^{4} \mathrm{~g} \mathrm{~cm}^{-3}$ which correspond to a sound velocity $v_{\mathrm{s}} / c=4.45 \times 10^{-2}$ or $v_{\mathrm{s}}=13350 \mathrm{~km} \mathrm{~s}^{-1}$ and a mass loss rate $\dot{m}=5.1 \times 10^{13} \mathrm{~g} \mathrm{~cm}^{-2} \mathrm{~s}^{-1}$. The neutrino heating and cooling terms are detailed in Fig. 7. The major contribution to the heating comes from neutrino captures on nucleons while neutrino emission by nucleons and annihilation of electron-positron pairs have comparable effects on the cooling. We have then abandoned the assumption of constant neutrino temperature which was
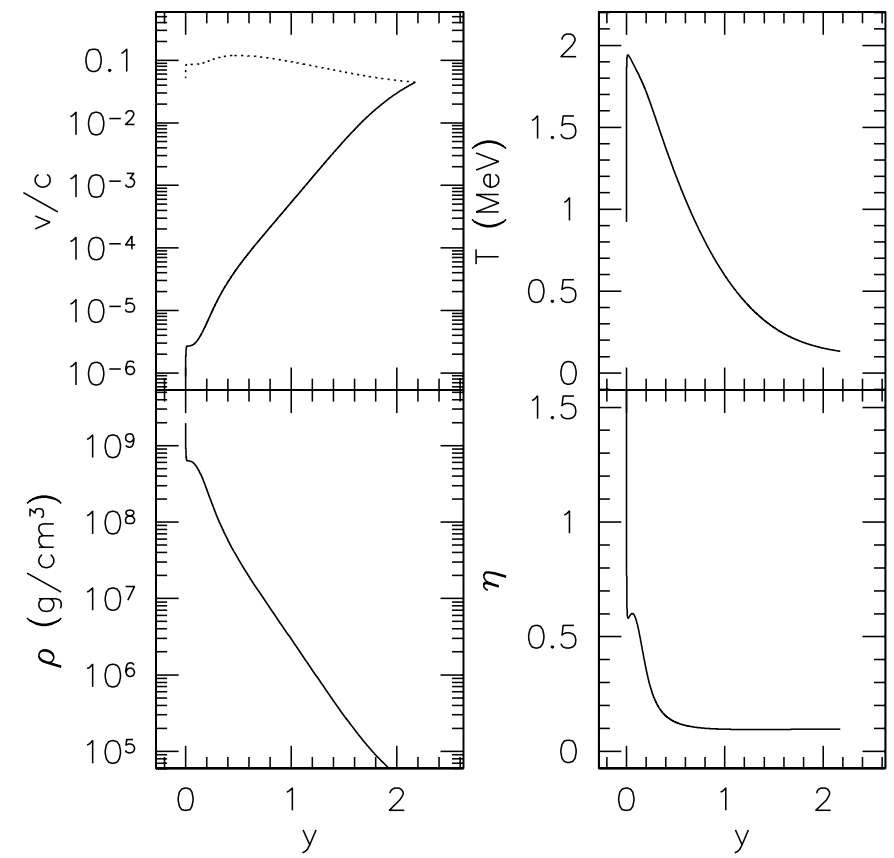

Fig. 6. Same as Fig. 3 for an optically thick disk.

essentially made for a rapid calculation of the integrals in the neutrino heating terms. When $T_{\nu}$ depends on the radial distance in the disk, the calculation of $\dot{q}_{\nu \bar{\nu}}$ becomes very time consuming. Since $\dot{q}_{\nu \bar{\nu}}$ is not the dominant neutrino heating term as long as $T_{\nu} \lesssim 10 \mathrm{MeV}$ we have neglected its contribution for the remainder of this paper. For example, in our reference model the mass loss rate is decreased from 5.1 to $4.9 \times 10^{13} \mathrm{~g} \mathrm{~cm}^{-2} \mathrm{~s}^{-1}$ if $\dot{q}_{\nu \bar{\nu}}$ is not included (i.e. a reduction of $4.5 \%$ ). Without $\dot{q}_{\nu \bar{\nu}}$, a non constant neutrino temperature can be easily implemented. We adopted for $T_{\nu}(r)$ relation (5) with $T_{*}=2 \mathrm{MeV}$ and the resulting mass loss rate is then $\dot{m}=3.8 \times$ $10^{13} \mathrm{~g} \mathrm{~cm}^{-2} \mathrm{~s}^{-1}$.

When $M_{\mathrm{BH}}, T_{\nu}, \theta$ or the position $r$ of the foot of the line are varied, the mass loss rate behaves as in the optically thin case

$$
\begin{aligned}
\dot{m}(x) \approx & 3.8 \times 10^{13} \mu_{\mathrm{BH}}\left[\frac{T_{\nu}(x)}{2 \mathrm{MeV}}\right]^{10} f[x, \theta(x)] \mathrm{g} \mathrm{cm}^{-2} \mathrm{~s}^{-1} \\
\approx & 3.8 \times 10^{13} \mu_{\mathrm{BH}}\left[\frac{T_{*}}{2 \mathrm{MeV}}\right]^{10}\left(\frac{r_{*}}{r}\right)^{15 / 2} \\
& \times\left(\frac{1-\sqrt{\frac{r_{\mathrm{in}}}{r}}}{1-\sqrt{\frac{r_{\mathrm{in}}}{r_{*}}}}\right)^{5 / 2} f[x, \theta(x)] \mathrm{g} \mathrm{cm}^{-2} \mathrm{~s}^{-1}
\end{aligned}
$$

The mass loss rate has been represented in Fig. 8 for $T_{*}=$ $2 \mathrm{MeV}, r_{*}=4 r_{\mathrm{h}}, r_{\mathrm{in}}=3 r_{\mathrm{h}}, r_{\mathrm{out}}=10 r_{\mathrm{h}}$ and the same field geometries already considered in Sect. 4.1.

\subsection{Analytical solution}

To obtain an analytical expression for the mass loss rate we have simplified the original wind problem by making several additional assumptions. We have first 


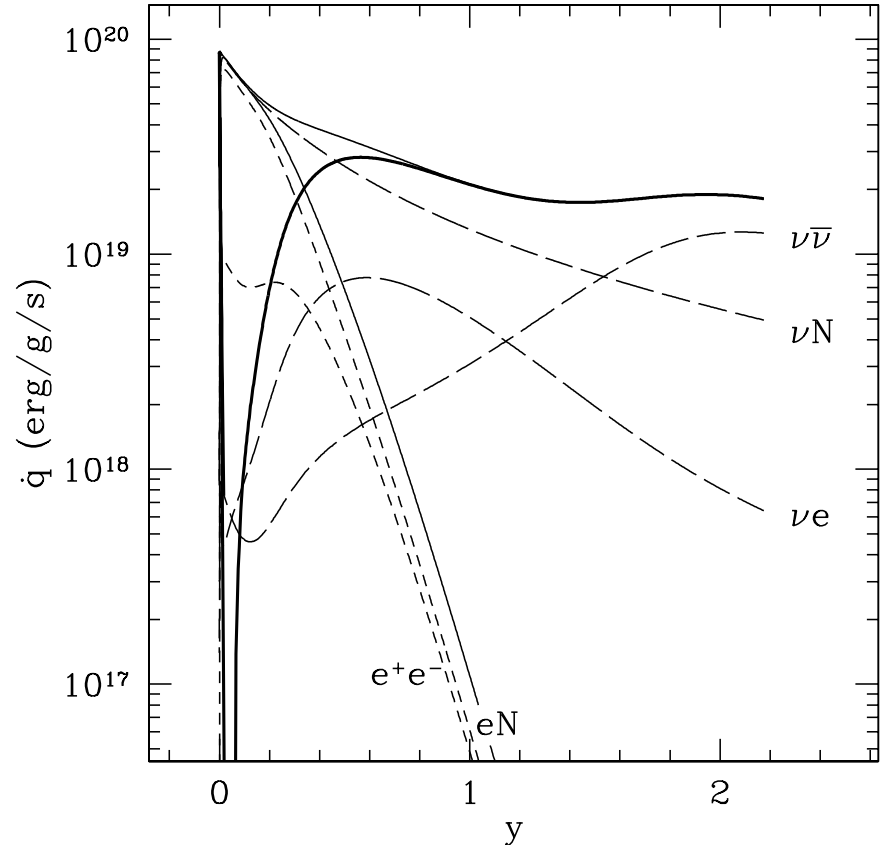

Fig. 7. Neutrino heating and cooling contributions. The long and short dashed lines respectively represent individual heating and cooling processes; $\nu \mathrm{N}$ : neutrino captures on nucleons; $\nu$ e: neutrino captures on electrons; $\nu \bar{\nu}$ : neutrino-antineutrino annihilation; eN: neutrino emission by nucleons; $\mathrm{e}^{+} \mathrm{e}^{-}$: annihilation of electron-positron pairs. The full thin lines represent the total of the heating $(\nu \mathrm{N}+\nu \mathrm{e}+\nu \bar{\nu})$ and cooling $\left(\mathrm{eN}+\mathrm{e}^{+} \mathrm{e}^{-}\right)$processes. The full thick line is the sum of all contributions.

considered that wind material is not too far from hydrostatic equilibrium even at the sonic point (in practice, the ratio $v \frac{\mathrm{d} v}{\mathrm{~d} r} / \gamma$ becomes larger than unity at $\left.\frac{y_{\mathrm{s}}-y}{y_{\mathrm{s}}} \sim 5 \%\right)$. We have also supposed that the pressure is dominated by the contribution of relativistic particles, which is equivalent to $\eta \ll 1$ since

$\frac{P_{\mathrm{N}}}{P_{\mathrm{r}}} \simeq 0.5 \frac{\eta}{Y_{\mathrm{e}}}$

In practice $\eta \simeq 0.1$ in our numerical solutions, except near the disk surface but we have nevertheless adopted $P \simeq$ $P_{\mathrm{r}} \propto T^{4}$ everywhere (we take $P_{\mathrm{r}}=\tilde{a} T^{4}$ with $\tilde{a}$ being given by Eq. (18) with $\eta=0.1$ ). Finally, we have located the sonic point at $y_{\mathrm{s}}=y_{1}$ where $\gamma(y)=0$. We then write the temperature

$T=T_{\mathrm{s}}\left(\frac{\rho}{\rho_{\mathrm{s}}}\right)^{1 / 3}(1+\tau)$

where the $\tau$ function is zero at the sonic point. From the sonic point down to the disk, as $\eta \propto \rho / T^{3}$ first remains nearly constant (see Figs. 3 and 6 ) $\tau$ stays close to zero. It then becomes negative when $\eta$ increases in the vicinity of the disk. With these assumptions the wind equations can be rewritten

$\rho v s=\dot{m}$

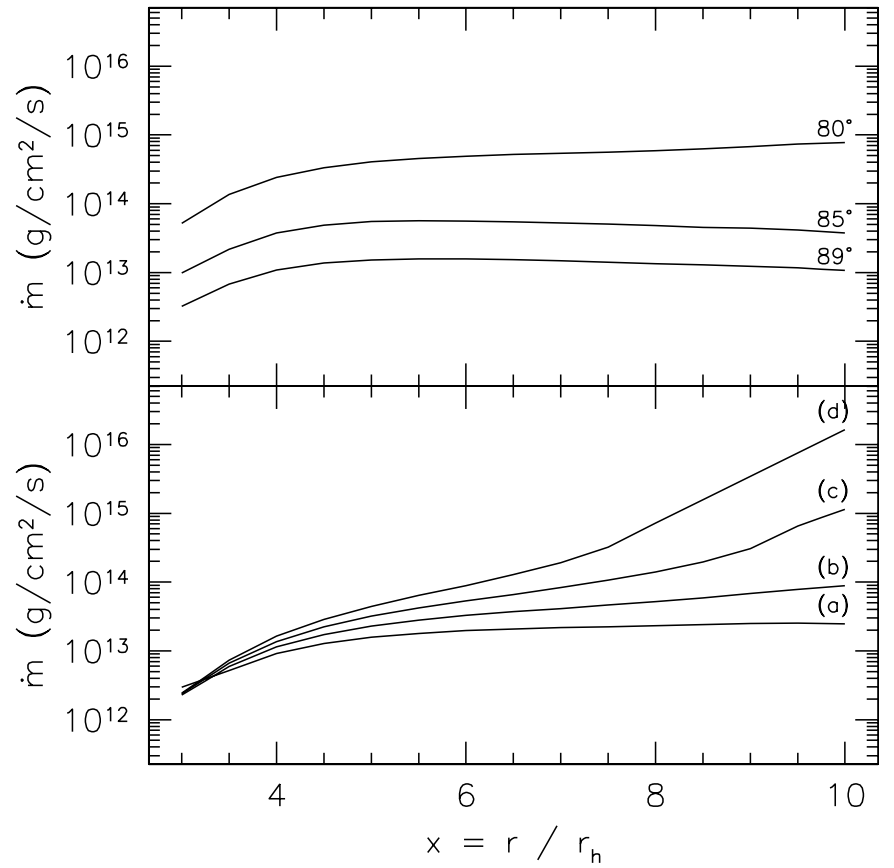

Fig. 8. Same as Fig. 5 for an optically thick disk.

$$
\begin{aligned}
& 4 \frac{P}{\rho} \frac{\mathrm{d} \log T}{\mathrm{~d} y}=\gamma r=\frac{\mathrm{d} \Phi}{\mathrm{d} y} \\
& 4 \frac{P}{\rho} \frac{\mathrm{d} \log (1+\tau)}{\mathrm{d} y}=\frac{\dot{q} r}{3 v}=\frac{\rho s \dot{q} r}{3 \dot{m}} .
\end{aligned}
$$

The solution of Eq. (43) gives

$T_{0}-T=\frac{1}{4 \tilde{a} \Theta} \int_{0}^{y} \frac{1}{(1+\tau)^{3}} \frac{\mathrm{d} \Phi}{\mathrm{d} y} \mathrm{~d} y$

where $\Theta=T_{\mathrm{s}}^{3} / \rho_{\mathrm{s}}$ and $T_{0}$ is the temperature in the plane of the disk. Taking $\tau=0$ everywhere leads to the approximate solution

$T_{0}-T \simeq \frac{1}{4 \tilde{a} \Theta}\left[\Phi(y)-\Phi_{0}\right]$

with $\Phi_{0}=\Phi(y=0)$. If we moreover suppose that $T_{\mathrm{s}} \ll T_{0}$ we get

$t(y)=\frac{T}{T_{0}} \simeq \frac{\Phi_{\mathrm{s}}-\Phi(y)}{\Phi_{\mathrm{s}}-\Phi_{0}}$

and

$\Theta \simeq \frac{\Phi_{\mathrm{s}}-\Phi_{0}}{4 \tilde{a} T_{0}}=\frac{\Delta \Phi}{4 \tilde{a} T_{0}}$

with $\phi_{\mathrm{s}}=\Phi\left(y_{\mathrm{s}}\right)=\Phi\left(y_{1}\right)$. In Fig. 3 the approximate value of the temperature given by Eq. (47) is compared to the exact solution obtained in Sect. 4.1. It can be seen that the agreement is quite satisfactory for $y<1$. We then transform Eq. (44) using relation (41), the definition (47) of $t(y)$ and writing $\dot{q}(y)$ as

$\dot{q}(y)=Q T_{0}^{6}\left[g(y)-t^{6}(y)\right]$

where $g(y)=1$ for an optically thin disk with uniform heating. In an optically thick disk we only consider the 
heating due to neutrino captures on nucleons and $g(y)$ is the geometrical integral appearing in Eq. (31). We obtain

$\frac{2}{3} \Theta^{2} \frac{\mathrm{d}(1+\tau)^{6}}{\mathrm{~d} y}=\frac{s(y) Q r}{3 \dot{m}} T_{0}^{8}\left\{t^{2}(y)\left[g(y)-t^{6}(y)\right]\right\}$.

Integrating this equation from the disk to the sonic point yields

$\dot{m}=\frac{Q r}{2 \tilde{a} \Theta^{2}\left[1-\left(1+\tau_{0}\right)^{6}\right]} T_{0}^{8} \mathcal{I}=\frac{8 \tilde{a} Q r}{\Delta \Phi^{2}-h_{0}^{2}} T_{0}^{10} \mathcal{I}$

where the integral

$\mathcal{I}=\int_{0}^{y_{1}} s(y) t^{2}(y)\left[g(y)-t^{6}(y)\right] \mathrm{d} y$

can be directly computed from Eqs. (47), (12) and (15). To obtain Eq. (51) we have used the value (48) of $\Theta$ and

$\left(1+\tau_{0}\right)^{3}=\frac{T_{0}}{\rho_{0} \Theta}=\frac{4 \tilde{a} T_{0}^{4}}{\rho_{0} \Delta \Phi}=\frac{h_{0}}{\Delta \Phi}$

all quantities with a zero subscript being taken in the plane of the disk. The analytical formula (51) reproduces the $T_{0}^{10}$ dependence of $\dot{m}$ and diverges when $h_{0}=\Delta \Phi$, i.e. when the Bernouilli function is equal to zero at the disk surface. The mass loss rate is also proportional to the mass of the black hole at constant $x=r / r_{\mathrm{h}}$. We have tested the accuracy of the analytical expression by computing $\dot{m}$ for the reference model with uniform heating (optically thin disk). We get $\dot{m}=3.9 \times 10^{14} \mathrm{~g} \mathrm{~cm}^{-2} \mathrm{~s}^{-1}$ in reasonable agreement with the numerical result obtained in Sect. 4.1.

\section{Estimates of the wind Lorentz factor}

In the simple model considered in this paper we do not follow the acceleration of the wind beyond the sonic point up to relativistic velocities. Therefore, the terminal Lorentz factor cannot be obtained in a self-consistent way. We simply expect that an average value of the terminal Lorentz factor will be given by

$\bar{\Gamma}=\frac{L_{\mathrm{w}}}{\dot{M} c^{2}}$

where $L_{\mathrm{w}}$ is the power injected into the wind and $\dot{M}$ is the total mass loss from the disk

$\dot{M}=\int_{r_{\text {in }}}^{r_{\text {out }}} \dot{m} 2 \pi r \mathrm{~d} r$.

The mass loss per unit surface of the disk has been obtained in the previous section

$\dot{m}(x)=\dot{m}_{0} \mu_{\mathrm{BH}}\left[\frac{T_{\mathrm{d}}(x)}{2 \mathrm{MeV}}\right]^{10} f[x, \theta(x)]$

so that

$\dot{M}=3.4 \times 10^{12} \dot{m}_{0} \mu_{\mathrm{BH}}^{3} \int_{x_{\mathrm{in}}}^{x_{\mathrm{out}}}\left[\frac{T_{\mathrm{d}}(x)}{2 \mathrm{MeV}}\right]^{10} f[x, \theta(x)] x \mathrm{~d} x$.

We have computed $\dot{M}$ for $x_{\text {in }}=3$ and $x_{\text {out }}=10$, both for optically thin and optically thick disks. In optically thin disks the temperature is given by Eq. (8) and $\dot{m}_{0}=$ $2.33 \times 10^{14} \mathrm{~g} \mathrm{~cm}^{-2} \mathrm{~s}^{-1}$. We have then

$\dot{M}=5.1 \times 10^{28} \mu_{\mathrm{BH}}\left(\frac{\alpha}{0.01}\right)^{2} F_{\text {geo }} \mathrm{g} \mathrm{s}^{-1}$

with

$F_{\text {geo }}=\int_{x_{\text {in }}}^{x_{\text {out }}} \frac{f[x, \theta(x)]}{x^{2}} \mathrm{~d} x$.

For an optically thick disk the temperature of the neutrinosphere is given by Eq. (5) and $\dot{m}_{0}=3.8 \times$ $10^{13} \mathrm{~g} \mathrm{~cm}^{-2} \mathrm{~s}^{-1}$ so that

$\dot{M}=7.9 \times 10^{28} \mu_{\mathrm{BH}}^{3}\left(\frac{T_{*}}{2 \mathrm{MeV}}\right)^{10} F_{\text {geo }} \mathrm{g} \mathrm{s}^{-1}$

with

$F_{\text {geo }}=\int_{x_{\text {in }}}^{x_{\text {out }}}\left(\frac{4}{x}\right)^{6.5}\left(1-\sqrt{\frac{3}{x}}\right)^{5 / 2} f[x, \theta(x)] \mathrm{d} x$.

We now introduce the ratio $\beta$ of the wind power to the disk neutrino luminosity

$L_{\mathrm{w}}=\beta L_{\nu}=\beta \int_{r_{\mathrm{in}}}^{r_{\mathrm{out}}} \frac{7}{8} \sigma T_{\nu}^{4}(r) 2 \pi r \mathrm{~d} r$

which yields the following estimate of $T_{*}$

$T_{*} \simeq 2 \mu_{\mathrm{BH}}^{-1 / 2}\left(\frac{L_{52}}{\beta_{5}}\right)^{1 / 4} \mathrm{MeV}$

where $L_{52}=L_{\mathrm{w}} / 10^{52} \operatorname{ergs}^{-1}$ and $\beta_{5}=\beta / 5$. A large $\beta$ corresponds to a disk able to transfer most of its energy in the Poynting flux with limited dissipation and heating. From Eqs. (58) and (63) we finally get

$\dot{M}=7.9 \times 10^{28} \mu_{\mathrm{BH}}^{-2}\left(\frac{L_{52}}{\beta_{5}}\right)^{5 / 2} F_{\text {geo }} \mathrm{g} \mathrm{s}^{-1}$.

We are now in a position to estimate the average Lorentz factor of the wind using Eq. (54). The results in the optically thin and optically thick cases are given respectively by

$$
\begin{aligned}
& \bar{\Gamma}=\frac{220}{F_{\text {geo }}} \mu_{\mathrm{BH}}^{-1} L_{52}\left(\frac{\alpha}{0.01}\right)^{-2} \text { opt. thin case } \\
& =\frac{140}{F_{\text {geo }}} \mu_{\mathrm{BH}}^{2} L_{52}^{-3 / 2} \beta_{5}^{5 / 2} \quad \text { opt. thick case }
\end{aligned}
$$

and the values of the geometric integrals are listed in Table 1 .

\section{Discussion and conclusions}

From Eq. (65) and the values of the geometric integrals in Table 1 it appears that the wind emitted from the inner disk can reach large Lorentz factors $(\Gamma>100)$ only under quite restrictive conditions on the disk temperature and field geometry. The disk temperature depends on the viscosity parameter $\alpha$ (in the optically thin case) and on 
Table 1. Values of the geometric integral $F_{\text {geo }}$ for an optically thin and an optically thick disk and the field geometries considered in Sect. 4. The corresponding values of $\bar{\Gamma}$ are computed using Eq. (65) with $\mu_{\mathrm{BH}}=1, L_{52}=1$ and $\alpha=0.01$ in the optically thin case and $\mu_{\mathrm{BH}}=1, L_{52}=1$ and $\beta=5$ in the optically thick case.

\begin{tabular}{lcccc}
\hline \hline Geometry & \multicolumn{2}{c}{ Opt. thin disk } & \multicolumn{2}{c}{ Opt. thick disk } \\
\hline & $F_{\text {geo }}$ & $\bar{\Gamma}$ & $F_{\text {geo }}$ & $\bar{\Gamma}$ \\
$\theta=80^{\circ}$ & 9.0 & 24 & 1.1 & 130 \\
$\theta=85^{\circ}$ & 1.1 & 200 & $9.2 \times 10^{-2}$ & 1500 \\
$\theta=89^{\circ}$ & 0.36 & 610 & $2.6 \times 10^{-2}$ & 5400 \\
$\theta=90^{\circ}-0.5^{\circ}(x-3)$ & 0.55 & 400 & $4.0 \times 10^{-2}$ & 3500 \\
$\theta=90^{\circ}-1 .^{\circ}(x-3)$ & 1.3 & 170 & $9.1 \times 10^{-2}$ & 1500 \\
$\theta=90^{\circ}-1.5^{\circ}(x-3)$ & $>5.8$ & $<38$ & 0.42 & 340 \\
$\theta=90^{\circ}-2 .^{\circ}(x-3)$ & $>200$ & $\approx 1$ & $>3.3$ & $<43$ \\
\hline
\end{tabular}

the value of $\beta$ (in the optically thick case). These two quantities which are quite uncertain unfortunately enter the expression of $\bar{\Gamma}$ with respective powers 2 and 2.5. The extreme sensitivity of $\bar{\Gamma}$ to the disk temperature also amplifies all errors and uncertainties in the evaluation of $T_{\mathrm{d}}$. For example, the simple analytical expression of $T_{\mathrm{d}}$ (Eq. (8)) used in the optically thin case does not depend on the accretion rate to the black hole while detailed calculations show that $T_{\mathrm{d}}$ slightly increases with $\dot{M}$ (Popham et al. 1999). A consequence of Eq. (8) is that $\bar{\Gamma} \propto L$ but just the opposite behavior (decreasing $\bar{\Gamma}$ with increasing $L)$ would be expected if $T_{\mathrm{d}} \propto \dot{M}^{x}$ with $x>0.1$. It is moreover even not clear that the $\alpha$-prescription remains appropriate in the context of strongly magnetized disks.

The Lorentz factor is also extremely sensitive to the field geometry: to escape from the disk, the material has to be heated so that its energy becomes large enough to cross the potential well. The height of this barrier decreases rapidly when the field lines are more inclined, leading to an increasing mass flux. As shown by our analytical solution of the wind equations, there is even a critical inclination where the mass flux diverges. This happens when the potential barrier is so shallow that the initial enthalpy of the material in the disk allows it to freely escape along the field lines, even without additional heating. For this reason quasi vertical field lines are required to prevent baryonic pollution from growing dramatically and slight changes of the inclination angle lead to large variations of the Lorentz factor. Between $\theta=90$ and $80^{\circ}, \bar{\Gamma}$ approximately behaves as $\theta^{30}$ ! A reduction by $1^{\circ}$ of the inclination angle therefore decreases $\bar{\Gamma}$ by a factor of about 1.5 .

Our incomplete description of the black hole and its environment prevents us from making any accurate prediction of the wind Lorentz factor. The numerical values of the mass loss rate given above have been obtained with a large set of simplifying assumptions. We present them to illustrate general tendencies which we believe are robust and can be used to evaluate the ability of realistic models to produce relativistic outflows. When the disk is thick or slim the temperature distribution and the heating or cooling processes will be different from the assumptions we have made. Similarly, the disk + black hole magnetosphere can be expected to have a very complex geometry and to be rapidly variable. The results of our toy model extreme sensitivity of the mass loss rate to the disk temperature $\left(\dot{m} \propto T_{\mathrm{d}}^{10}\right)$ and field geometry - indicate that high terminal Lorentz factors can be reached only under severe constraints: disk temperature $T_{\mathrm{d}}$ not largely exceeding $2 \mathrm{MeV}$ and presence of at least a few field lines pointing directly away from the disk in the vertical direction.

If such conditions can be satisfied so that the wind can indeed become relativistic, it is then easy to understand that its baryonic load and hence its Lorentz factor can strongly vary on short time scales as a result of fluctuations of the disk temperature or field geometry. Conversely, if the outflow remains non relativistic $(\bar{\Gamma} \sim 1)$ because the disk is too hot or the field lines deviate too much from the vertical, the burst must be produced by the Blandford-Znajek effect alone with no contribution from accretion energy. The dense wind emitted from the disk can then have both a beneficial and negative effect on the central relativistic jet. It can probably help to confine the jet but can also represent a risk of baryonic pollution via Kelvin-Helmholtz instabilities or magnetic reconnection at the jet-wind interface.

Acknowledgements. We thank the anonymous referee for his careful reading of the manuscript and thoughtful comments.

\section{References}

Artemova, I. V., Bjoernsson, G., \& Novikov, I. D. 1996, ApJ, 461, 565

Baring, M. G., \& Harding, A. K. 1997, ApJ, 491, 663

Bethe, H. A. 1993, ApJ, 412, 192

Bethe, H. A., Applegate, J. H., \& Brown, G. E. 1980, ApJ, 241,343

Blandford, R. D., \& McKee, C. F. 1976, Phys. Fluids, 19, 1130

Blandford, R. D., \& Payne, D. G. 1982, MNRAS, 199, 883

Blandford, R. D., \& Znajek, R. L. 1977, MNRAS, 179, 433

Calder, A. C., Wang, E. Y. M., \& Swesty, F. D. 1999, Amer. Astron. Soc. Meet., 194, 11.602

Chevalier, R. A., \& Li, Z. 2000, ApJ, 536, 195

Daigne, F., \& Mochkovitch, R. 1998, MNRAS, 296, 275

Daigne, F., \& Mochkovitch, R. 2000, A\&A, 358, 1157

Davies, M. B., Benz, W., Piran, T., \& Thielemann, F. K. 1994, ApJ, 431, 742

Dermer, C. D., \& Mitman, K. E. 1999, ApJ, 513, L5

Djorgovski, et al. 2001, in Proc. IX Marcel Grossmann Meeting, ed. V. Gurzadyan, R. Jantzen, \& R. Ruffini (Singapore: World Scientific), in press [astro-ph/0106574]

Duncan, R. C., Shapiro, S. L., \& Wasserman, I. 1986, ApJ, 309, 141

Faber, J. A., Manor, J., \& Rasio, F. A. 2000, Amer. Astron. Soc. Meet., 196, 38.03

Galama, T. J., Vreeswijk, P. M., van Paradijs, J., et al. 1998, Nature, 395, 670

Hachisu, I. 1986, ApJS, 61, 479 
Herant, M., Benz, W., Hix, W. R., Fryer, C. L., \& Colgate, S. A. 1994, ApJ, 435, 339

Janka, H.-T., Eberl, T., Ruffert, M., \& Fryer, C. L. 1999, ApJ, 527, L39

Jaroszynski, M. 1993, Acta Astron., 43, 183

Klose, S., Stecklum, B., Masetti, N., et al. 2000, ApJ, 545, 271

Kobayashi, S., Piran, T., \& Sari, R. 1997, ApJ, 490, 92

MacFadyen, A. I., \& Woosley, S. E. 1999, ApJ, 524, 262

Meszaros, P., \& Rees, M. J. 1992a, MNRAS, 257, 29P

Meszaros, P., \& Rees, M. J. 1992b, ApJ, 397, 570

Meszaros, P., \& Rees, M. J. 1997, ApJ, 476, 232

Mochkovitch, R., Hernanz, M., Isern, J., \& Martin, X. 1993, Nature, 361, 236

Narayan, R., Paczynski, B., \& Piran, T. 1992, ApJ, 395, L83

Oohara, K., \& Nakamura, T. 1997, in Relativistic Gravitation and Gravitational Radiation, 309

Ostriker, J. P., \& Mark, J. W. 1968, ApJ, 151, 1075

Owens, A., Guainazzi, M., Oosterbroek, T., et al. 1998, A\&A, 339, L37

Paczynski, B. 1998, ApJ, 494, L45
Popham, R., Woosley, S. E., \& Fryer, C. 1999, ApJ, 518, 356

Preece, R. D., Briggs, M. S., Mallozzi, R. S., et al. 1998, ApJ, 506, L23

Qian, Y.-Z., \& Woosley, S. E. 1996, ApJ, 471, 331

Rees, M. J., \& Meszaros, P. 1994, ApJ, 430, L93

Rhoads, J. 1997, in Fourth Huntsville Gamma-Ray Burst Symposium, held 15-20 September, 1997, 02

Rosswog, S., Davies, M. B., Thielemann, F.-K., \& Piran, T. 2000, A\&A, 360, 171

Ruffert, M., Janka, H., Takahashi, K., \& Schaefer, G. 1997, A\&A, 319, 122

Ruffert, M., \& Janka, H.-T. 1999, A\&A, 344, 573

Ruffert, M., Janka, H.-T., \& Schaefer, G. 1996, A\&A, 311, 532

Sari, R., Piran, T., \& Narayan, R. 1998, ApJ, 497, L17

van Paradijs, J., Groot, P. J., Galama, T., et al. 1997, Nature, 386,686

Waxman, E., Kulkarni, S. R., \& Frail, D. A. 1998, ApJ, 497, 288

Woosley, S. E. 1993, ApJ, 405, 273 